\title{
Iterative Reconstruction of SPECT Images using Adaptive Multi-Level Refinement
}

\author{
Hanno Schumacher ${ }^{1,3}$, Stefan Heldmann ${ }^{1}$, Eldad Haber ${ }^{2}$, Bernd Fischer ${ }^{1}$ \\ ${ }^{1}$ University of Lübeck, Institute of Mathematics, Wallstr. 40, 23560 Lübeck, Germany \\ ${ }^{2}$ Emory University, Mathematics and Computer Science, Atlanta, GA 30322, USA \\ ${ }^{3} \mathrm{MiE}$ GmbH, Hauptstr. 112, 23845 Seth, Germany \\ schumaha@math.uni-luebeck.de
}

\begin{abstract}
We present a novel method for iterative reconstruction of high resolution images. Our method is based on the observation that constant regions in an image can be represented at much lower resolution than region with fine details. Therefore, we combine adaptive refinement based on quadtrees with iterative reconstruction to reduce the computational costs. In our experiments we found a speed up factor of approximately two compared to a standard multi-level method.
\end{abstract}

\section{Introduction}

Imaging systems are important tools in today's clinical routine. Tomographic images as, e.g., MRI, CT, PET, or SPECT images, have become standard for diagnosis and clinical testing. However, due to existing techniques, image reconstruction from tomographic data is still an open and active research field.

Here, we focus on iterative reconstruction methods particularly for SPECT. Such algorithms are able to compute high resolution high quality images and they are particularly useful when dealing with noisy data. Nevertheless, computing high quality images comes not for free. The complexity of such algorithms depends on the desired (high) target resolution. Typical dimensions are $128^{3}$, $256^{3}$, or $512^{3}$ involving huge amount of data and thus lead to a non-neglectable computational effort.

Our new approach reduces the computational complexity without reducing the desired resolution. The basic idea is that regions of (almost) constant intensities can be represented at a very low resolution. To this end, we combine iterative reconstruction with adaptive multi-level refinement. Starting from a very low resolution, we locally refine regions where we expect details in the image while other regions with, e.g., only background information, stay coarse. Due to their simplicity and the rectangular pixel-structure of image, we propose quad- (2D) and octrees (3D) for the local refinement [1].

The ideas of representing images with local low and high resolutions as well as adaptive refinement are not new. These techniques are well known and have already been used in many other fields, too. Recent examples particularly incorporating quad-/octrees include image registration $[2,3,4]$, computer graphics 
[5], or inverse problems [6]. A similar approach for image reconstruction using a fixed non-uniform mesh generated from external knowledge is presented in [7]. However, to our best knowledge adaptive multi-level refinement has not been used for image reconstruction, yet.

The paper is organized as follows. In section 2 we state the image reconstruction problem and describe our new method. Subsequently, we present a real SPECT data and an academic example in section 3. Finally we discuss the results in section 4 .

\section{Materials and Methods}

Here we focus on reconstruction of SPECT images [8]. Therefore, emitted radiation of an injected radioactive contrast agent is measured at different position around the object. Then, the measured projection data $d$ is used to reconstructed an image $f$ such that the image $f$ represents the distribution of the contrast agent. Formally, this leads to the problem

$$
\mathcal{A} f=d \quad \text { s.t. } \quad f \geq 0
$$

with a linear projection operator $\mathcal{A}$ describing the SPECT measurement. The constraint $f \geq 0$ is required to guarantee a positive distribution since negative concentrations are not physical. It is well known that (1) is an ill-posed problem $[9,10]$. Therefore, additional constraints and/or regularization is needed. Furthermore, real measured data $d$ comes with non-neglectable noise such that solving $\mathcal{A} f=d$ for $f$ exactly will not produce wanted results. Therefore, instead of trying to solve (1) directly, we consider the optimization problem

$$
\operatorname{minimize} \quad \mathcal{J}(f)=\frac{1}{2}\|\mathcal{A} f-d\|_{L_{2}}^{2}+\alpha \mathcal{R}(f) \quad \text { s.t. } \quad f \geq 0
$$

Here, $\frac{1}{2}\|\mathcal{A} f-d\|_{L_{2}}^{2}$ forces fitting the data $d$ and $\mathcal{R}$ is a regularizer addressing the ill-posedness and the noise in the data. Typically, $\mathcal{R}$ forces smoothness of the image $f$ such that the parameter $\alpha>0$ weights the influence of the regularizer versus the (noisy) data fit. In particular, here we choose

$$
\mathcal{R}(f)=\frac{1}{2} \sum_{i=1}^{n}\left\|\partial_{i} f\right\|_{L_{2}}^{2}
$$

where $n$ is the dimension of the image $f(n=2$ or $n=3)$.

To compute a numerical solution we first discretize (2) on a given quad- or octree. We proceed analog to $[1,2,3,6]$. Quad- and octrees are an efficient way to compress structured data. To make the idea clear, let us consider a regular discretization of a image composed of pixels/voxels with uniform size. For the quad-/octree we combine square blocks of pixels and represent each of theses blocks by a single large pixel, i.e., lower resolution. See Figures 1,2 for 
2D quadtree examples. Thus, we discretize the image on cell-centers of the given quad-/octree such that the discretized problem reads

$$
\operatorname{minimize} \quad \mathbf{J}(\mathbf{f})=\frac{1}{2}\|\mathbf{A f}-\mathbf{d}\|_{2}^{2}+\frac{\alpha}{2} \mathbf{f}^{\top} \mathbf{B}^{\top} \mathbf{B} \mathbf{f} \quad \text { s.t. } \quad \mathbf{f} \geq 0
$$

where the matrix $\mathbf{A}$ is a discretization of the $\mathcal{A}$ and the $\mathbf{f}^{\top} \mathbf{B}^{\top} \mathbf{B} \mathbf{f}$ is a finite difference approximations to the regularizer $\mathcal{R}(f)$ [5].

Our overall method is an adaptive multi-level scheme. We start with discretizing the problem on a coarse regular grid say 8 by 8 and compute a minimizer of (4). Subsequently, we locally refine our discretization due to some criteria explained below and compute a minimizer of (4) discretized on the new grid. Then, we locally refine again, solve the problem on the new grid and so on. We proceed until the finest cells in our discretization have reached the target resolution.

To compute a minimizer of (4) on a fixed level we use Newton's method with projecting each iterate to the constraints $\mathbf{f} \geq 0$. The method is similar to the gradient-projection algorithm described in [11] except that for fast convergence we take a Newton search direction instead of the negative gradient. Therefore, in each step we have to solve a large linear system. Here we spend the main computational efforts and this is also the point where we get the major benefit from our method by reducing the number of unknowns. Our experiments show that we gain a speedup of a factor 2 compared to a standard method.

Our local refinement strategy is based on the general idea that constant regions of an image can be represented with low resolution than regions with rapidly changing gray values, i.e., edges. To this end, we consider the gradient of the image and do a refinement where it is large. Since we do not have an analytic gradient, we compute a finite difference approximation $[5,6]$. Let $\mathbf{g}_{j}$ be an approximation to the image gradient on the $j$-th cell of our current grid such that $\mathbf{g}_{j} \approx \nabla f\left(\operatorname{cell}_{j}\right)$. Then we subdivide the $j$-th cell into four sub-cells if $\left\|\mathbf{g}_{j}\right\|_{\infty}>\gamma$ where $\gamma>0$ is a chosen parameter.

\section{Results}

We present two 2D examples where we compare our adaptive method with a standard uniform refinement. In the first academic example, we reconstruct a Shepp-Logan-Phantom ( $256^{2}$ pixels, max. gray value 255$)$ where the artificial projection data was generated from an ideal noise-free image. The aim of this experiment is to demonstrate that we are able to reconstruct high quality images on quite sparse quadtrees. For the second example we reconstruct images $\left(128^{2}\right.$ pixels, max. gray value 800) from real SPECT data. Here, we demonstrate the use of our method for real data with noise. In both experiments we started our multi-level method from a coarse 8 by 8 grid and the regularization parameter $\alpha$ was set to $10^{-3}$.

For the Shepp-Logan-Phantom we simulated 120 SPECT projections with 256 pixels each. We evaluated several choices for the refinement parameter $\gamma$. The resulting images and quadtrees are presented in Figure 1. As expected, the 
Fig. 1. Results for the Shepp-Logan-Phantom
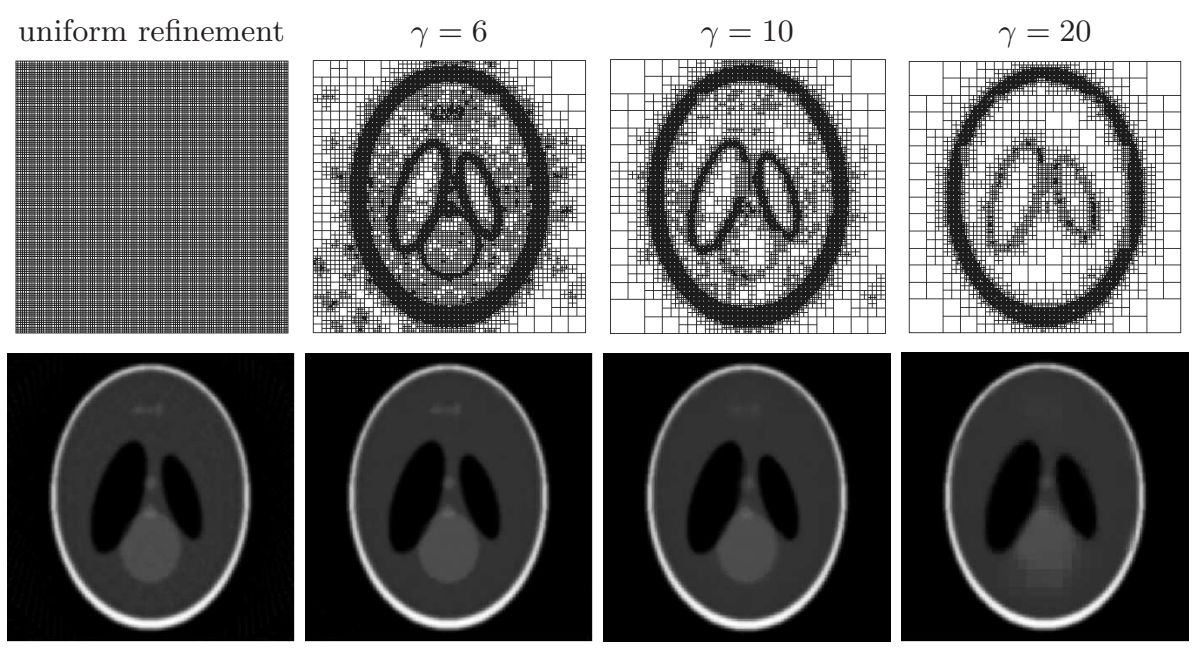

results show that with increasing $\gamma$ the resulting quadtrees become more sparse and the number of pixels is drastically reduced. Nevertheless, the visual quality of the images remains reasonable even for very large values of $\gamma$.

In our real data example, 120 projections of the Benchmark-Jaszczak-Phantom were measured with a SPECT system from the MiE company in Seth, Germany. In contrast to the academic example, here we have to deal with noise that is included in the data. However, we are able to compute images on sparse quadtrees that are visually indistinguishable from the result when using a standard uniform refinement (Fig. 2).

In both experiments we successfully apply our adaptive refinement strategy that drastically reduces computational efforts. Basically, we found that we only need $30-50 \%$ of the pixels resulting an overall speedup factor of approximately 2. The computational results for both experiments are presented in Table 1.
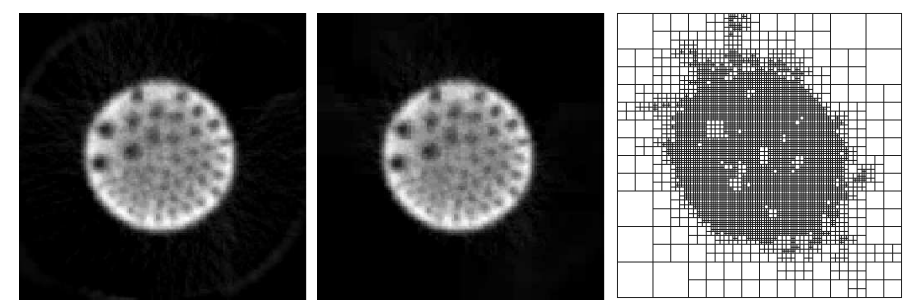

Fig. 2. Results for the Benchmark-Jaszczak-Phantom. Left result for uniform refinement, middle + right result after adaptive refinement $(\gamma=40)$ 
Table 1. Computational results for the finest level computed in Matlab

\begin{tabular}{lccccc}
\hline Data & refinement & \#iter & average time per iter & \#pixels & resolution \\
\hline Shepp-Logan & uniform & 6 & $5.39 \mathrm{sec}$ & 65,536 & $256^{2}$ \\
Shepp-Logan & $\gamma=6$ & 3 & $2.96 \mathrm{sec}$ & 22,198 & $256^{2}$ \\
Shepp-Logan & $\gamma=10$ & 4 & $2.22 \mathrm{sec}$ & 16,732 & $256^{2}$ \\
Shepp-Logan & $\gamma=20$ & 4 & $1.52 \mathrm{sec}$ & 11,749 & $256^{2}$ \\
Real-data & uniform & 4 & $1.25 \mathrm{sec}$ & 16,384 & $128^{2}$ \\
Real-data & $\gamma=40$ & 4 & $0.60 \mathrm{sec}$ & 5,782 & $128^{2}$ \\
\hline
\end{tabular}

\section{Discussion}

We have presented a novel multi-level reconstruction scheme using an adaptive refinement strategy based on quadtrees. The method is able to produce high quality images at the same level of detail as standard methods while significantly reducing the computational costs. Moreover, no external knowledge about the image content is used. In our experiments we found a speedup factor of roughly 2 . Our examples demonstrate the potential of the new method and its successful use with real data having noise.

The presented results are basically $2 \mathrm{D}$ and can be used for slice-wise reconstruction of 3D data. However, in future work the approach shall be extended to fully $3 \mathrm{D}$, i.e., by using $3 \mathrm{D}$ octrees instead of $2 \mathrm{D}$ quadtrees. Furthermore, we aim for an automatic estimate of the refinement parameter $\gamma$.

\section{References}

1. Samet H. The Design and Analysis of Spatial Data Structures. Boston, MA, USA: Addison-Wesley Longman Publishing Co., Inc.; 1990.

2. Haber E, Heldmann S, Modersitzki J. An OcTree method for parametric image registration. SIAM J Sci Comp. 2006;Accepted.

3. Haber E, Heldmann S, Modersitzki J. Adaptive mesh refinement for nonparametric image registration. SIAM J Sci Comp. 2007;Submitted.

4. Szeliski R, Lavallée S. Matching 3-D anatomical surfaces with non-rigid deformation using Octree-splines. Proc SPIE. 1993;2031.

5. Losasso F, Gibou F, Fedkiw R. Simulating water and smoke with an octree data structure. ACM Trans Graph. 2004;23(4):457-62.

6. Haber E, Heldmann S, Ascher U. Adaptive finite volume methods for distributed non-smooth parameter identification. Inverse Problems. 2007;23:1659-76.

7. Brankov JG, Yang Y, Wernick MN. Tomographic image reconstruction based on a content-adaptive mesh model. IEEE Trans Med Imaging. 2004;23(2):202-12.

8. Groch MW, Erwin WD. SPECT in the Year 2000: Basic principles. J Nucl Med Technol. 2000;28(4):233-44.

9. Natterer F, Wübbeling F. Mathematical Methods in Image Reconstruction. Philadelphia: Society for Industrial and Applied Mathematics; 2001.

10. Bruyant P. Analytic and iterative reconstruction algorithms in SPECT. J Nucl Med. 2002;43(10):1343-58.

11. Nocedal J, Wright SJ. Numerical Optimization. Springer; 1999. 